\title{
Exercícios aeróbicos aquáticos em indivíduos com Paralisia Cerebral: Revisão Sistemática
}

\author{
Aquatic aerobic exercises in individuals with Cerebral Palsy: \\ Sistematic Review
}

\author{
Ejercicios aeróbico acuático en personas con Parálisis \\ Cerebral: Revisión Sistematica
}

\author{
Letícia de Oliveira Bonifácio ${ }^{1}$, Juliana Aparecida Bette Sanches ${ }^{1}$, \\ Douglas Martins Braga ${ }^{2}$, Caio Roberto Aparecido de Paschoal Castro ${ }^{3}$
}

1.Aluna do Aperfeiçoamento em fisioterapia nas disfunções neurológicas da criança e do adulto (AACD). São Paulo-SP, Brasil.

2.Supervisor do setor de fisioterapia aquática da Associação de Assistência à Criança Deficiente AACD/Ibirapuera. São Paulo-SP, Brasil.

3. Fisioterapeuta do setor de fisioterapia aquática da Associação de Assistência à Criança Deficiente AACD/Ibirapuera. São Paulo-SP, Brasil.

\begin{abstract}
Resumo
Objetivo. Observar os principais efeitos dos exercícios aeróbicos aquáticos em indivíduos com paralisia cerebral. Método. Foi realizada uma revisão de literatura entre março de 2020 e novembro de 2020, através das bases de dados MEDLINE (Pubmed), PEDro, Cochrane Library, LILACS e Scielo. A revisão foi registrada na PROSPERO com o número CRD42020207445. Os artigos encontrados na busca foram armazenados no software Rayyan QCRI. Após o armazenamento, a seleção dos estudos foi realizada por dois pesquisadores de forma independente e cegada, através da avaliação dos títulos e resumos. Após a inclusão dos estudos, foi realizada uma análise narrativa e descritiva. Resultados. Foram encontrados 122 artigos na busca inicial nas bases de dados. Destes, 2 dos artigos foram encontrados de forma duplicada, restando 120 estudos. Na etapa da seleção por pares independentes, de forma cegada, foram incluídos 4 artigos e 91 artigos foram excluídos. Os pesquisadores entraram em conflito de decisão em 15 artigos e ficaram na dúvida de inclusão em 10 artigos. Os 25 artigos foram lidos na íntegra e revisados por um terceiro pesquisador, sendo incluído mais 1 artigo, totalizando 5 artigos selecionados para a revisão. Conclusão. Através desta revisão observamos que os exercícios aeróbicos aquáticos podem promover benefícios para crianças e adolescentes com paralisia cerebral. Porém, mais estudos e ensaios clínicos são necessários para comprovar a eficácia deste tipo de intervenção e seus benefícios específicos.
\end{abstract}

Unitermos. Paralisia cerebral; hidroterapia; exercícios aeróbicos; condicionamento físico

\begin{abstract}
Objective. To observe the main effects of aquatic aerobic exercises in individuals with cerebral palsy. Method. A systematic review was carried out between March 2020 and November 2020, using the MEDLINE (Pubmed), PEDro, Cochrane Library, LILACS and Scielo databases. The review was registered with PROSPERO under number CRD42020207445. The articles found in the search were stored in the Rayyan QCRI software. After storage, the selection of studies was carried out by two researchers independently and blindly, through the evaluation of titles and abstracts. After the inclusion of the studies, a narrative and descriptive analysis was carried out. Results. 122 articles were found in the initial search in the databases. Of these, 2 of the articles were found in duplicate, with 120 studies remaining. In the stage of selection by independent peers, blindly, 4 articles were included, and 91 articles were excluded. The researchers were in a conflict of decision in 15 articles and were in doubt about inclusion in 10 articles. The 25 articles were read in full and reviewed by a third researcher, including 1 more article, totaling 5 articles selected for review. Conclusion. Through this review we observed that aquatic aerobic exercises can promote benefits for children and adolescents with cerebral
\end{abstract}


palsy. However, further studies and clinical trials are needed to prove the effectiveness of this type of intervention and its specific benefits.

Keywords. Cerebral palsy; hydrotherapy; aerobic exercises; physical fitness

\begin{abstract}
Resumen
Objetivo. Observar los principales efectos de los ejercicios aeróbicos acuáticos en individuos con parálisis cerebral. Método. Se realizó una revisión sistemática entre marzo de 2020 y noviembre de 2020, utilizando las bases de datos MEDLINE (Pubmed), PEDro, Cochrane Library, LILACS y Scielo. La revisión se registró con PROSPERO con el número CRD42020207445. Los artículos encontrados en la búsqueda se almacenaron en el software Rayyan QCRI. Después del almacenamiento, la selección de los estudios fue realizada por dos investigadores de forma independiente y ciega, mediante la evaluación de títulos y resúmenes. Luego de la inclusión de los estudios, se realizó un análisis narrativo y descriptivo. Resultados. se encontraron 122 artículos en la búsqueda inicial en las bases de datos. De estos, 2 de los artículos se encontraron por duplicado, quedando 120 estudios. En la etapa de selección ciega por pares, se incluyeron 4 artículos y se excluyeron 91 artículos. Los investigadores estaban en un conflicto de decisión en 15 artículos y tenían dudas sobre la inclusión en 10 artículos. Los 25 artículos fueron leídos en su totalidad y revisados por un tercer investigador, incluido 1 artículo más, totalizando 5 artículos seleccionados para revisión. Conclusión. Mediante esta revisión observamos que los ejercicios aeróbicos acuáticos pueden promover beneficios para niños y adolescentes con parálisis cerebral. Sin embargo, se necesitan más estudios y ensayos clínicos para demostrar la eficacia de este tipo de intervención y sus beneficios específicos.
\end{abstract}

Palabras clave. Parálisis cerebral; hidroterapia; ejercicios aeróbicos; condicionamiento físico

Trabalho realizado na Associação de Assistência à Criança Deficiente, AACD/Ibirapuera. São Paulo-SP, Brasil.

\title{
INTRODUÇÃO
}

A Paralisia cerebral (PC) é definida por ser um conjunto de desordens motoras e sensoriais, decorrentes de lesões no encéfalo imaturo ${ }^{1}$. Essas lesões resultam em alterações neuromotoras persistentes, porém não progressivas, com variação do tônus, da postura e do movimento ${ }^{1,2}$. A sua prevalência é de 1,5 a 3,8:1.000 nascidos vivos no mundo ${ }^{3}$. O Gross Motor Function Classification System (GMFCS) classifica estes indivíduos conforme as atividades que eles executam com frequência no seu dia-a-dia ${ }^{1,4}$. A classificação é dividida em cinco níveis (I, II, III, IV e V), sendo inversamente proporcional à função motora grossa ${ }^{5-8}$. 
Independente dos níveis do GMFCS, os pacientes apresentam alterações de tônus muscular, diminuição de força muscular, alterações de equilíbrio, mobilidade e independência 9 . Todas estas alterações ocasionam uma cascata de acometimentos, repercutindo na diminuição da capacidade aeróbia ${ }^{10}$.

A diminuição da capacidade aeróbia desencadeia um ciclo de sedentarismo e descondicionamento nestes indivíduos, elevando o risco de comorbidades como obesidade, cardiopatias e diminuição da qualidade de vida ${ }^{11}$.

Indivíduos com PC podem apresentar dificuldades para a execução dos exercícios máximos e submáximos, por conta de sobrecargas articulares, alterações posturais, amplitudes de movimentos limitadas e quadros álgicos ${ }^{12,13}$. Portanto, uma das alternativas para a realização dos exercícios aeróbicos é a realização no ambiente aquático ${ }^{14}$.

O ambiente aquático promove benefícios através dos princípios físicos e termodinâmicos da água ${ }^{14}$. O indivíduo em imersão sofre alterações fisiológicas, como, aumento do débito cardíaco, diminuição da resistência vascular periférica, diminuição da frequência cardíaca e consequente facilitação do retorno venoso, por conta da pressão hidrostática e temperatura aquecida da água ${ }^{15}$. Estas alterações são diretamente proporcionais ao aumento do nível de imersão ${ }^{15}$. A temperatura da água entre 33 e 36 graus Celsius auxilia na diminuição do quadro álgico e promove um relaxamento muscular que pode facilitar a movimentação ativa das regiões álgicas ${ }^{16}$. Além disso, por 
conta do empuxo, a realização de movimentos antigravitacionais fica facilitada, aumentando a amplitude de movimento e diminuindo a sobrecarga articular ${ }^{15,16}$. A viscosidade aumenta o tempo para a realização de ajustes posturais, promovendo maior segurança para a realização dos exercícios ${ }^{16}$.

Sabe-se que o exercício aeróbico realizado no ambiente aquático aumenta a circulação sanguínea cerebral, principalmente das artérias cerebrais anterior e média, que são responsáveis pela irrigação de áreas sensório-motoras e núcleos inibitórios ${ }^{16}$. Exercícios aeróbicos realizados na piscina melhoram a capacidade cardiorrespiratória, a cognição, funcionalidade e independência de pessoas com alterações neurológicas, além da melhora na qualidade de vida de indivíduos com desordens neurológicas ${ }^{17}$.

Com isso, o objetivo da atual revisão é observar os principais efeitos do treino aeróbico aquático em indivíduos com PC.

\section{MÉTODO}

Foi realizada uma revisão bibliográfica entre março de 2020 e novembro de 2020, sem restrições de idiomas e datas de publicações, através das bases de dados MEDLINE (Pubmed), PEDro, Cochrane Library, LILACS e Scielo. A revisão foi registrada na PROSPERO com o número CRD42020207445.

Para a busca foram utilizados os descritores (Mesh Terms) "cerebral palsy"; "hydrotherapy"; "hydrotherapies", 
"aerobic exercise", "physical endurance", "fitness" e termos utilizados frequentemente na literatura, como "aquatic therapy", "aquatic exercises" e "water-based exercises". Todos os termos foram utilizados em inglês e seus representantes na língua portuguesa, atrelados pelos descritores booleanos AND ou OR.

Os artigos encontrados na busca foram armazenados no software Rayyan QCRI. Após o armazenamento, a seleção dos estudos foi realizada por dois pesquisadores de forma independente e cegada, através da avaliação dos títulos e resumos. No caso de dúvidas na inclusão, os pesquisadores leram os artigos na íntegra. Nos casos de divergências entre a inclusão ou exclusão, entre os dois pesquisadores, um terceiro avaliador realizou a análise e decidiu a inclusão ou a exclusão destes estudos. Os artigos incluídos foram lidos na íntegra.

Os artigos incluídos na revisão deveriam seguir os seguintes critérios de inclusão, respondendo ao acrônimo PICOT (People; Intervention; Comparison; Outcome; Type): a) "P" População: indivíduos com Paralisia Cerebral; b) "I" Intervenção: exercícios aeróbicos aquáticos; c)" $C$ " Comparação: qualquer população ou intervenção; d) "O" Desfechos: Todos desfechos encontrados; e)"T" Tipos de estudos: intervencionais.

Foram excluídos os artigos que descreviam somente $o$ protocolo da intervenção.

Após a inclusão dos estudos, foi realizada uma análise narrativa e descritiva. 


\section{RESULTADOS}

Foram encontrados 122 artigos na busca inicial nas bases de dados. Destes, 2 dos artigos foram encontrados de forma duplicada, restando 120 estudos.

$\mathrm{Na}$ etapa da seleção por pares independentes, de forma cegada, foram incluídos 4 artigos e 91 artigos foram excluídos. Os pesquisadores entraram em conflito de decisão em 15 artigos e ficaram na dúvida de inclusão em 10 artigos. Os conflitos de decisão e dúvidas na inclusão ocorreram devido à falta de clareza da metodologia nos resumos ou nos títulos dos estudos. Os 25 artigos foram lidos na íntegra e revisados por um terceiro pesquisador, sendo incluído mais 1 artigo, totalizando 5 artigos selecionados para a revisão. A Figura 1 demostra o fluxograma do estudo.

Figura 1. Fluxograma do estudo.

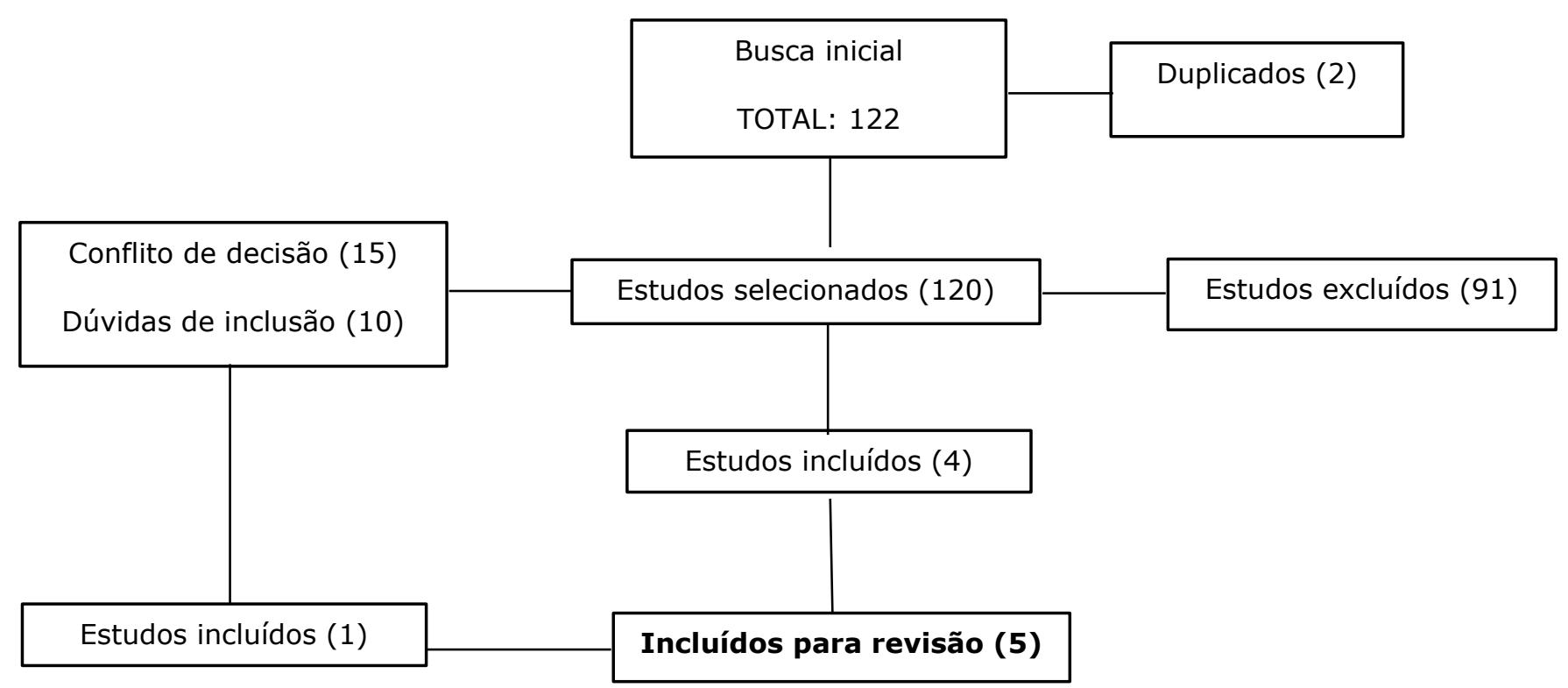


O estudo de Depiazzi et al. 202014 investigou a viabilidade do treinamento aquático intervalado de alta intensidade para adolescentes (12-17 anos) com PC classificados como níveis II no GMFCS. Os participantes passaram por uma avaliação de pico de performance e somente os participantes que concluíram a avaliação, foram incluídos no estudo. Estes, foram aleatorizados em dois grupos; grupo de cuidados usuais (controle) e grupo de exercícios aquáticos intervalados de alta intensidade. O treinamento aquático de alta intensidade foi realizado duas vezes na semana, por um período de 10 semanas, composto por 10 exercícios de 60 segundos com intervalos de 60 segundos de descanso, por 40 minutos. O exercício de alta intensidade foi definido como a obtenção da frequência cardíaca máxima $>80 \%$, medida por telemetria.

O estudo de Ballaz et al. $2011^{18}$ avaliou o efeito e a viabilidade de um programa de treinamento aquático em grupo de 10 semanas sobre a eficiência da marcha em adolescentes com PC. Os participantes usavam um monitor de frequência cardíaca para avaliar a intensidade das sessões. A terapia foi composta por 10 minutos de aquecimento, 15 minutos de corrida, 5 minutos de aquecimento e 15 minutos de atividades aquáticas (polo aquático ou vôlei aquático). Após o programa de treinamento foi observada uma redução significativa do índice de gasto energético e da frequência cardíaca durante a marcha, demonstrando que o treinamento aquático em grupo pode beneficiar a marcha de adolescentes com PC. 
Dimitrijevic et al. $2012^{19}$ realizaram um estudo que buscou verificar o efeito da fisioterapia aquática na função motora grossa, tônus muscular e resistência cardiorrespiratória em crianças com PC níveis II e III. A intervenção foi composta por 2 sessões de fisioterapia aquática, com duração de 50 minutos cada, durante 12 semanas. As sessões foram constituídas de exercícios de flutuação e marcha dentro da piscina. O estudo mostrou que o programa de exercícios aquáticos melhorou a função motora grossa, reduziu a espasticidade e aumentou a resistência cardiorrespiratória em crianças com PC espástica.

No estudo de Fragala-Pinkham et al. 201320, as intervenções foram realizadas em uma piscina com temperatura entre 32 e 34 graus Celsius e foi composta por exercícios aeróbicos. A frequência cardíaca alvo foi estabelecida como 70 a $80 \%$ da frequência cardíaca máxima. Ao final da intervenção verificou-se melhora nas funções motoras grossas mesmo após um mês da intervenção. A distância percorrida no teste de caminhada de 6 minutos foi significativamente maior somente no momento após o protocolo.

Retarekar et al. 200921, avaliou os efeitos dos exercícios aeróbios aquáticos sobre a função motora grossa, gasto energético e performance ocupacional de uma criança com PC. As sessões foram divididas em 3 fases: A1 coleta de dados ( 6 semanas), $B$ intervenção e $A 2$ onde foi realizado $O$ acompanhamento de 13 semanas após intervenção. A fase $B$ foi realizada três vezes por semana durante 12 semanas, 
em uma piscina com 32 a 34 graus Celsius. Os exercícios foram baseados em aquecimento (5 minutos), exercícios aeróbicos (30-40 minutos), desaquecimento (5 minutos), totalizando 40-50 minutos, com frequência cardíaca de treinamento de 50 a $80 \%$ da frequência cardíaca de reserva. Verificou-se que a criança obteve aumento na mobilidade nos ambientes domésticos e comunitários, principalmente na fase A2, o mesmo ocorreu no componente de satisfação, sugerindo satisfação dos pais a partir dos resultados obtidos pelo COPM. Houve um aumento no escore do GMFM de 2,71 pontos que permaneceu ao longo das 13 semanas após a intervenção. Houve aumento de $27 \%$ na distância percorrida durante o teste de caminha de 6 minutos, totalizando um aumento de $9 \mathrm{~m} / \mathrm{min}$ na velocidade e a distância de caminhada em 56 metros, no entanto, esse resultado regrediu progressivamente na fase A2. Já no Índice de Gasto Energético foi observado melhora na eficiência e velocidade da marcha na fase $\mathrm{B}$ de 3,12.

O Quadro 1 descreve os estudos incluídos na atual revisão, demonstrando os dados do tipo do estudo, idade dos participantes, GMFCS, desfechos e instrumentos de medida, tipo de intervenção e conclusão dos estudos.

O Quadro 2 descreve o tipo de estudo dos artigos incluídos nesta revisão. 
Quadro 1. Descrição dos estudos incluídos- falta colocar a legenda das siglas do quadro.

\begin{tabular}{|c|c|c|c|c|c|c|}
\hline Autor & $\begin{array}{l}\text { Tipo de } \\
\text { estudo }\end{array}$ & $\begin{array}{c}\text { Idade } \\
\text { Amostra }\end{array}$ & Gmfcs & $\begin{array}{l}\text { Instrumento } \\
\text { s de medida }\end{array}$ & Intervenção & Conclusão \\
\hline $\begin{array}{l}\text { Retarekar et } \\
\text { al. } 2009^{21}\end{array}$ & $\begin{array}{l}\text { Estudo de } \\
\text { caso }\end{array}$ & $\begin{array}{c}5 \text { anos } \\
1 \\
\text { participante }\end{array}$ & III & $\begin{array}{c}\text { - GMFM } \\
\text { - COPM } \\
\text { - TC6MIN } \\
\text { - INDICE DE } \\
\text { GASTO } \\
\text { ENERGETICO }\end{array}$ & $\begin{array}{c}36 \text { sessões de } 40-50 \\
\text { minutos } \\
\begin{array}{c}3 \times / \text { semana por } 12 \\
\text { semanas }\end{array} \\
\begin{array}{c}\text { Água entre } 32 \text { e } 34 \\
\text { graus }\end{array}\end{array}$ & $\begin{array}{c}\text { Foi observado } \\
\text { melhora da } \\
\text { função motora } \\
\text { grossa e } \\
\text { performance } \\
\text { ocupacional, } \\
\text { aumento da } \\
\text { velocidade da } \\
\text { marcha e } \\
\text { diminuição do } \\
\text { gasto energético. }\end{array}$ \\
\hline $\begin{array}{l}\text { Fragala- } \\
\text { Pinkham et } \\
\text { al. } 2013^{20}\end{array}$ & $\begin{array}{l}\text { Série de } \\
\text { casos }\end{array}$ & $\begin{array}{c}\text { 6-15 anos } \\
8 \\
\text { participantes }\end{array}$ & I e III & $\begin{array}{c}\text { - GMFM (D e } \\
\text { E) } \\
\text { - TC6MIN }\end{array}$ & $\begin{array}{l}14 \text { semanas } \\
2 x / \text { semana } \\
60 \text { minutos }\end{array}$ & $\begin{array}{l}\text { O estudo } \\
\text { verificou melhora } \\
\text { da função } \\
\text { motoras grossa e } \\
\text { distância } \\
\text { percorrida na } \\
\text { marcha. }\end{array}$ \\
\hline $\begin{array}{l}\text { Depiazzi et } \\
\text { al. } 2020^{14}\end{array}$ & $\begin{array}{c}\text { Estudo } \\
\text { piloto: } \\
\text { ensaio } \\
\text { clínico } \\
\text { aleatorizad } \\
\text { o } \\
\text { controlado }\end{array}$ & $\begin{array}{l}\text { Média de } 14 \\
\text { anos } \\
12 \\
\text { participantes } \\
\text { (6 grupo } \\
\text { controle e } 6 \\
\text { grupo } \\
\text { intervenção) }\end{array}$ & II & $\begin{array}{l}\text { - VO } \mathrm{V}_{2} \text { Peak } \\
\text { - BAPQ } \\
\text { - COPM } \\
\text { - PedsQL } \\
\text { - Densidade } \\
\text { óssea }\end{array}$ & $\begin{array}{l}10 \text { semanas } \\
2 x / \text { semana } \\
60 \text { minutos }\end{array}$ & $\begin{array}{l}\text { O estudo concluiu } \\
\text { que o exercício } \\
\text { intervalado de } \\
\text { alta intensidade é } \\
\text { aplicável para } \\
\text { indivíduos com } \\
\text { PC. }\end{array}$ \\
\hline $\begin{array}{l}\text { Ballaz } \\
2011^{18}\end{array}$ & $\begin{array}{c}\text { Série de } \\
\text { casos }\end{array}$ & $\begin{array}{c}\text { 14-21 anos } \\
12 \\
\text { participantes }\end{array}$ & $\begin{array}{l}\text { I, II, III } \\
\text { e IV }\end{array}$ & $\begin{array}{l}\text { - Índice de } \\
\text { gasto } \\
\text { energético } \\
\text { - GMFM (D e } \\
\text { D) } \\
\text { - Variáves da } \\
\text { marcha }\end{array}$ & $\begin{array}{l}20 \text { sessões } \\
2 x / \text { semana } \\
45 \text { minutos }\end{array}$ & $\begin{array}{l}\text { Foi observada } \\
\text { redução do gasto } \\
\text { energético e da } \\
\text { FC durante a } \\
\text { marcha. }\end{array}$ \\
\hline $\begin{array}{l}\text { Dimitrijevic } \\
\text { et al. } 2012^{19}\end{array}$ & $\begin{array}{c}\text { Série de } \\
\text { casos }\end{array}$ & $\begin{array}{c}\text { 6-12 anos } \\
19 \\
\text { participantes }\end{array}$ & $\begin{array}{l}\text { I, II, III } \\
\text { e IV }\end{array}$ & $\begin{array}{l}\text { - GMFM } \\
\text { - MAS } \\
\text { - FC } \\
\text { - } \mathrm{VO}_{2} \text { máx }\end{array}$ & $\begin{array}{l}12 \text { semanas } \\
2 x / \text { semana } \\
60 \text { minutos }\end{array}$ & $\begin{array}{c}\text { O estudo } \\
\text { demonstrou } \\
\text { melhora na } \\
\text { função motora } \\
\text { grossa, } \\
\text { diminuição da } \\
\text { espasticidade e } \\
\text { melhora do } \\
\text { condicionamento } \\
\text { físico de } \\
\text { indivíduos com } \\
\text { PC. }\end{array}$ \\
\hline
\end{tabular}

GMFM - Gross Motor Function Classification System; COPM - Canadian Occupational Performance Measure; TC6MIN - Teste de caminhada de 6 minutos; $\mathrm{VO}_{2 \text { Peak }}$ - Pico de consume de oxigênio; $\mathrm{VO}_{2 \text { máx }}$ Consumo máximo de oxigênio; PedsQL - Pediatric Quality of Life Inventory; MAS - Modified Ashworth Scale; BAPQ - Broad Autism Phenotype Questionnaire; FC - Frequência cardíaca. 
Quadro 2. Tipos de estudos incluídos na revisão.

\begin{tabular}{|l|l|}
\hline Autor & Tipo de estudo \\
\hline Retarekar et al. $2009^{21}$ & Estudo de caso \\
\hline Fragala-Pinkham et al. $2013^{20}$ & Série de casos \\
\hline Depiazzi et al. $2020^{14}$ & Estudo piloto: ensaio clínico aleatorizado controlado \\
\hline Ballaz $2011^{18}$ & Série de casos \\
\hline Dimitrijevic et al. $2012^{19}$ & Série de casos \\
\hline
\end{tabular}

\section{DISCUSSÃO}

Crianças com PC diparéticas espásticas apresentam disfunções que às predispõem a níveis mais baixos de consumo de oxigênio, por conta das alterações biomecânicas da caixa torácica, alterações do tônus e postura. Levando em consideração as respostas fisiológicas típicas geradas pelos exercícios aeróbicos, sabe-se que é possível o aumento e melhora da aptidão aeróbica, atividade e participação do indivíduo, onde foi evidenciada a partir das comparações das escalas propostas pelos autores.

Retarekar et al. $2009^{21}$ observa que habilidades como levantar-se e deambular, entre outras habilidades motoras, são mantidas durante e após término da intervenção, mas isso não ocorreu com a velocidade de marcha e no gasto energético de um indivíduo com PC. Fragala-Pinkham et al. $2013^{20}$ relataram achados que corroboram com este estudo, verificando que a melhora adquirida na capacidade aeróbia de crianças e adolescentes com PC não permaneceram após o final do estudo. Portanto, os autores pontuaram a importância da continuidade de atividades aquáticas ou 
exercícios aquáticos adaptados e programas exercícios solos com treinamento aeróbico para a durabilidade desses ganhos.

No estudo de Ballaz et al. $2011^{18}$ os participantes classificados como GMFCS III e IV, obtiveram menor índice de gasto energético, embora tenham realizado exercícios mais intensos por menos tempo, quando comparados aos participantes classificados como GMFCS I e II. Com isso podemos inferir que a redução da capacidade aeróbia pode ocorrer em indivíduos com PC de todos os níveis funcionais. As adaptações cardiorrespiratórias, possivelmente, colaboraram para a melhora da resistência aos exercícios, através do treinamento aeróbico no estudo de Retarekar et al. $2009^{21}$.

A literatura ressalta que indivíduos com PC tem alto risco de desenvolvimento de doenças relacionadas ao estilo de vida sedentária e essas estimativas são maiores para os indivíduos menos funcionais, por conta do déficit de mobilidade e a dificuldade da continuidade das atividades na vida adulta ${ }^{22}$. No estudo de Ballaz et al. $2011^{18}$ indivíduos com maior comprometimento funcional, consumiram menos energia do que indivíduos mais funcionais, nas atividades propostas no ambiente aquático. Porém, estes exercícios foram de maior intensidade e menor tempo, fazendo com que mecanismos anaeróbios fossem mais exigidos, do que no grupo dos pacientes mais funcionais.

Os programas aquáticos devem ser incorporados junto as terapias convencionais ao tratamento de crianças com PC, 
sendo considerado um modo contínuo e essencial de tratamento, a fim de garantir melhorias da função motora grossa à longo prazo ${ }^{23}$. Estas considerações estão de acordo com os estudos de Ratarekar et al. $2009^{21}$ e Fragala-Pinkham et al. 201320, que enfatizam a importância da continuidade de programas de exercícios após o término das intervenções propostas em cada um dos estudos.

Crianças com PC que participam de um programa de treinamento de exercícios aeróbicos demonstram melhorias nas medidas fisiológicas da capacidade aeróbia ${ }^{24}$. As evidências de que crianças com PC são capazes de realizar este tipo de intervenção, traz informações positivas e abrem novas perspectivas para o tratamento desta população e devem servir como um incentivo para futuros estudos que estabeleçam diretrizes de exercícios seguros, eficazes e específicos para crianças de diferentes idades e habilidades.

As intervenções dos programas de exercícios aeróbicos analisados nesta revisão não foram homogêneas, fato este que limita a comprovação da eficácia desta intervenção.

Novos estudos que comprovem a eficácia das intervenções de exercícios aeróbicos para crianças com PC necessitam ser realizados.

\section{CONCLUSÃO}

O exercício aeróbico aquático pode promover benefícios para a capacidade aeróbia e função motora grossa de crianças e adolescentes com PC. O desenvolvimento de ensaios clínicos controlados e aleatorizados é necessário 
para que se possa comprovar a eficácia e os benefícios desta intervenção.

\section{REFERÊNCIAS}

1.Rosenbaum PL, Palisano RJ, Bartlett DJ, Galuppi BE, Russell DJ. Development of the Gross Motor Function Classification System for cerebral palsy. Dev Med Child Neurol 2008;50:249-53. https://doi.org/10.1111/j.1469-8749.2008.02045.x

2.Silva RKA, Souto DO. Reabilitação dos membros inferiores na paralisia cerebral diplégica. Fisiot Bras 2020;21:104-13. https://doi.org/10.33233/fb.v21i1.2840

3. Ryan JM, Cassidy EE, Noorduyn SG, O'Connell NE. Exercise interventions for cerebral palsy. Cochrane Database Syst Rev 2017;6:CD011660.

https://doi.org/10.1002/14651858.CD011660.pub2

4.Bonomo LMM, Castro VC, Ferreira DM, Miyamoto ST. Hydrotherapy in the acquisition of the functionality of children with Cerebral Palsy. Rev Neurocienc 2007;15:125-30.

https://doi.org/10.34024/rnc.2007.v15.10293

5. Oliveira AIA, Golin MO, Cunha MCB. Applicability of the classification system of gross motor function (GMFCS) in cerebral palsy - review. Arq Bras Cienc Saúde 2010;35:200-24.

https://doi.org/10.7322/abcs.v35i3.85

6. Fajardo-López N, Moscoso-Alvarado F. Training aerobic capacity through the aquatic therapy in children with cerebral palsy type spastic diplegia. Rev Facultad Med 2013;61:365-71.

http://www.scielo.org.co/pdf/rfmun/v61n4/v61n4a5.pdf

7. Rocha AP, Afonso DRV, Morais RLDS. Relação entre desempenho funcional de crianças com paralisia cerebral e qualidade de vida relacionada à saúde de seus cuidadores. Fisiot Pesq 2008;15:292-7. https://doi.org/10.1590/S1809-29502008000300013

8.Dias ACB, Freitas JC, Formiga CKMR, Viana FP. (2010). Desempenho funcional de crianças com paralisia cerebral participantes de tratamento multidisciplinar. Fisiot Pesq 2010;17:225-29. https://doi.org/10.1590/S1809-29502010000300007

9.Schmitz FS, Stigger F. Atividades aquáticas em pacientes com paralisia cerebral: um olhar na perspectiva da fisioterapia. Rev Atenção Saúde 2014;12:78-89. https://doi.org/10.13037/rbcs.vol12n42.2428 10.Batista KG, Lopes PO, Serradilha SM, Souza GAF, Bella GP, Spuza RCT. Benefits of cardiorespiratory training in children or adolescents with cerebral palsy. Fisioter Mov 2010;33:201-9.

https://doi.org/10.1590/S0103-51502010000200004

11. Nova FASV. Atividade aquática e paralisia cerebral. Arq Mov 2012;8:69-84. https://doi.org/10.13037/rbcs.vol12n42.2428

12. Rogers A, Furler BL, Brinks S, Darrah J. A systematic review of the effectiveness of aerobic exercise interventions for children with 
cerebral palsy: an AACPDM. evidence report. Develop Med Chin Neurol 2008;50:808-14. https://doi.org/10.1111/j.1469-8749.2008.03134.x 13.Fragala-Pinkham M, Haley SM, O'Neil ME. Group aquatic aerobic exercise for children with disabilities. Develop Med Chil Neurol 2008;50:822-7. https://doi.org/10.1111/j.1469-8749.2008.03086.x 14.Depiazzi J, Smith N, Gibson N, Wilson A, Langdon K, Hill K. Aquatic high intensity interval training to improve aerobic capacity is feasible in adolescents with cerebral palsy: pilot randomised controlled trial. Clinic Rehabil 2021;35:222-31.

https://doi.org/10.1177/0269215520956499

15.Basmajian JV. Terapêutica por exercícios. 3. ed. São Paulo: Manole; 2000.

16.Becker BE, Cole AJ. Terapia aquática moderna. São Paulo: Manole; 1980.

17. Moura EW, Lima E, Borges J, Silva PAC. Fisioterapia: Aspectos Clínicos e Práticos da Reabilitação. 2. Ed. São Paulo: Artes Médicas; 2009.

18. Ballaz L, Plamondon S, Lemay M. Group aquatic training improves gait efficiency in adolescents with cerebral palsy. Disab Rehab 2011;33:1616-24. https://doi.org/10.3109/09638288.2010.541544

19.Dimitrijević L, Aleksandrović M, Madić $D$, Okičić T, Radovanović $D$, Daly $D$. The effect of aquatic intervention on the gross motor function and aquatic skills in children with cerebral palsy. J Hum Kinet 2012;32:167. https://doi.org/10.2478/v10078-012-0033-5

20.Fragala-Pinkham MA, Smith HJ, Lombard KA, Barlow C, O'Neil ME. Aquatic aerobic exercise for children with cerebral palsy: a pilot intervention study. Physiother Theory Pract 2014;30:69-78. https://doi.org/10.3109/09593985.2013.825825

21.Retarekar R, Fragala-Pinkham MA, Townsend EL. Effects of aquatic aerobic exercise for a child with cerebral palsy: single-subject design. Ped Phys Ther 2009;21:336-44. https://doi.org/10.1097/PEP.0b013e3181beb039

22. Cremer N, Hurvitz EA, Peterson MD. Multimorbidity in Middle-Aged Adults with Cerebral Palsy. Am J Med 2017;130:744.e9-15. https://doi.org/10.1016/j.amjmed.2016.11.044

23.Ballington SJ, Naidoo R. The carry-over effect of an aquatic-based intervention in children with cerebral palsy. Afric J Disab 2018;7:1-8. http://dx.doi.org/10.4102/ajod.v7i0.361

24.Rogers A, Furler BL, Brinks S, Darrah J. A systematic review of the effectiveness of aerobic exercise interventions for children with cerebral palsy: an AACPDM. evidence report. Develop Med Chil Neurol 2008; 50:808-14.

http://dx.doi.org/10.1111/j.1469-

8749.2008.03134.x 